\title{
Validity and Reliability of an Assessment Tool for the Screening of Neurotoxic Effects in Agricultural Workers in Chile
}

\author{
Boris Lucero ${ }^{D},{ }^{1}$ Paula A. Ceballos, ${ }^{2}$ María Teresa Muñoz-Quezada, ${ }^{1}$ Carolina Reynaldos, ${ }^{1}$ \\ Chiara Saracini, ${ }^{1}$ and Brittney Olivia Baumert ${ }^{3}$ \\ ${ }^{1}$ The Neuropsychology and Cognitive Neurosciences Research Center (CINPSI Neurocog), Faculty of Health Sciences, \\ Universidad Católica del Maule, Talca 3460000, Chile \\ ${ }^{2}$ Department of Nursery, Faculty of Health Sciences, Universidad Católica del Maule, Talca 3460000, Chile \\ ${ }^{3}$ Department of Environmental Health, Rollins School of Public Health, Emory University, Atlanta, GA 30322, USA
}

Correspondence should be addressed to Boris Lucero; balucero@gmail.com

Received 24 June 2019; Revised 15 September 2019; Accepted 27 September 2019; Published 30 October 2019

Guest Editor: Xudong Wang

Copyright (c) 2019 Boris Lucero et al. This is an open access article distributed under the Creative Commons Attribution License, which permits unrestricted use, distribution, and reproduction in any medium, provided the original work is properly cited.

There is a substantial use of pesticides within the agricultural industry of Chile, with neurotoxic effects through mechanisms of acetylcholinesterase inhibition. These pesticides result in deterioration in health, increasing the risk of diseases such as Parkinson's and Alzheimer's in highly exposed occupational population. To date, there are no brief assessment tools to monitor cognitive impairment in agricultural workers chronically exposed to these pesticides. Method. 234 agricultural workers and 305 nonagricultural workers were assessed two times (test-retest) through a brief tool which comprised three tests (clock-drawing test (CDT); frontal assessment battery (FAB); trail making tests (TMT) A and B). The full scale of WAIS-IV was administered as a gold standard to $18 \%$ of the sample of agricultural workers. Factor analysis was used to evaluate the factor structure, and validity and test-retest reliability were assessed concurrently. Results. Cronbach's alpha values were satisfactory or above $(>0.60)$. Test-retest correlations were all significantly correlated $(p<0.001)$. All the tests had a significant correlation with the full scale IQ score of WAIS-IV $(p<0.05)$. The Kaiser-Meyer-Olkin $(\mathrm{KMO})$ measure was 0.74 , and the Bartell sphericity test $=p<0.001$. Three factors explaining $61.62 \%$ of the variance were extracted. Two items of the FAB test were dropped of the final factor solution. Normative data transformed into percentile scores and stratified by age and educational level were obtained for Chilean agricultural workers. Conclusion. The brief assessment tool has adequate metric properties as a screening instrument. This allows for a simple administration test (10 to 15 minutes) that can potentially be used for the rapid monitoring of cognitive deterioration in the face of occupational exposure to pesticides in agricultural workers.

\section{Introduction}

Adverse health outcomes from pesticide-related exposure are a global issue impacting both industrialized and developing countries $[1,2]$. There is evidence that those most critically impacted by the adverse health implications from pesticide exposure are those in the occupational agricultural setting [3-8].

In Chile, the most frequently sold group of pesticides is the organophosphates (OP), specifically diazinon and chlorpyrifos [9]. The primary mode of action of OP pesticides is via the enzyme acetylcholinesterase [10]. The inhibition of acetylcholinesterase generates an excessive excitation of the muscarinic and nicotinic receptors of the nervous system, causing the acetylcholine neurotransmitter to overaccumulate in the cholinergic synapses. This eventually results in acute or chronic intoxication. Studies found that the neurotoxic effect of pesticides can provoke decreased cognitive performance in developing children and can be considered a precursor to an increased risk of degenerative diseases in chronically exposed workers in occupational settings $[3,11,12]$. In the last decades, increased attention has been paid internationally to the development and administration of tools which can evaluate the 
neurobehavioral and cognitive effects of the exposure to neurotoxic substances such as pesticides [5-7]. The administration of these kinds of tools to populations of agricultural workers exposed to chemicals has been developed for decades using methods derived from neuropsychology and experimental psychology. Usually, batteries of a series of behavioral tests have been used to evaluate the effects of neurotoxic pesticides, and the number of tests to be used in these batteries has increased over time $[7,10,13]$. One of the most important tools is the Neurobehavioral Core Test Battery (NCTB) [14]. It is composed of seven behavioral tools: Digit Symbol test, Digit Span test, Simple Reaction Time, Benton Visual Retention test, Santa Ana Dexterity test, Pursuit Aiming II, and the Mood Profile [15, 16]. Noteworthy, the selection of the tests composing the NCTB battery has been performed on the basic rule that these must be proven to be tools sensitive to the changes associated with exposure to chemicals. It means that the tests have shown to be effective in differentiating between groups of workers who had been exposed or not to chemicals, allowing the identification of neurotoxin effects. This implies that batteries also had to comply with the two basic psychometric properties of validity and reliability. In this regard, in 1999 [17], one of the main recommendations for NCTB was the need to establish construct validity in a wide range of different countries. In fact, it had been previously concluded that this battery could effectively assess only the adult population with twelve or more years of education and belonging to North America, Western Europe, and some parts of Asia, while it did not show the same reliability of use on people with less than nine years of education, being additionally discarded its effective use in populations different from the North American and European. In 2013, different aspects of the use of the battery and the selection criteria of the tests for the measurement of neurotoxic effects were discussed again [18]. For example, it was recommended that the elaboration of such type of battery should not include copyrighted tests. This was a relevant point, since most of the tests included in the NCTB were copyrighted; in fact, they should be acquired individually in order to be used legitimately. Instead of this, experts recommend including tests that are of public domain, such as the trail making test (TMT) [19]. A final conclusion regarding the tests is that they should be free of cultural influence. Experts furthermore agreed that it is always necessary to have a control group for comparison in epidemiological studies of neurotoxic exposures. Particularly in the case of the workers, it was pointed out that it was necessary to apply tests in a test and retest context, in order to establish an early monitoring of the occupationally exposed populations with respect to the neurotoxic effect on a follow-up logic. Finally, they concluded that it is necessary to develop a dedicated "screening" assessment tool made up of sensitive tests to be used in the field of human neurotoxicology [18]. Other authors pointed out that the neuropsychological tools that result in a greater effect size with respect to OP pesticides are those that measure working memory/attention, visual memory, psychomotor speed, executive function, and visuospatial ability [5]. Therefore, it can be concluded that the cognitive functions affected to a greater extent by the exposure to OP pesticides are nonverbal abilities, being found in crosssectional studies a slowdown of reaction time and deficits in the performance in short-term memory and executive function in the most severely affected individuals [5]. Moreover, a constant monitoring of the agricultural workers' neurocognitive functioning is necessary, due to the evidence that pesticides increase the risk for neurodegenerative diseases, such as Parkinson's [20] or dementia and Alzheimer's disease (AD), as it has been observed in longitudinal cohort studies [21].

Different types of neurotoxic disorders have been associated with chronic OP exposure: (1) cholinergic syndrome; (2) intermediate syndrome; (3) OP-induced polyneuropathy; (4) chronical exposure-related neuropsychiatric disorders. The first two disorders are generally detected in case of acute intoxication in the emergency care hospitals and are immediately treated and registered. However, the third and fourth kind of diseases need a detailed monitoring, for example, through a screening assessment tool, and their symptoms may appear later in the individual's life, with a progressive deterioration of the neurobehavioral performance, associated to permanent damages in the central nervous system (CNS), and cognitive deficits, including memory, concentration and learning, attention, information processing, and reaction times.

In Chile, there are few studies in the area collecting evidence of neurocognitive risk in the agricultural workers, the most complete and relevant being the one carried out in the Maule region [22]. In this study, full intelligence scales (Wechsler Adult Intelligence Scale, WAIS-IV) [23] were used to measure neurocognitive effects. The use of this kind of tools, although is advisable in terms of a more specific notion of the performance and the cognitive profile of test takers, implies a longer time of both test administration and further interpretation of the results. These "practical" features make it difficult to adopt them as screening and monitoring tests considering the amount of human and financial resources needed to perform the study, both from an organizational and individual point of view. Therefore, in the Chilean context, nationally validated tools (such as batteries of tests) able to show neurological problems in agricultural occupational populations which are at the same time sensitive and viable for a fast and easy administration are still missing. For this reason, it appears clear that it is necessary to establish an economic test, easy to apply and validated at a national level with agricultural occupational population for the early monitoring of the cognitive effects of exposure to acetylcholinesterase inhibitor pesticides. This test should be directly and specifically focused on the evaluation of these cognitive areas that have been recognized as more sensitive in the adverse effects on workers' health (reaction time, short-term memory, and executive functions), in order to perform an effective surveillance in the context of occupational exposure. Accordingly, the aim of this study is to validate a brief assessment tool for the monitoring of neurotoxic effects in agricultural workers exposed to pesticides, which can be used as a screening 


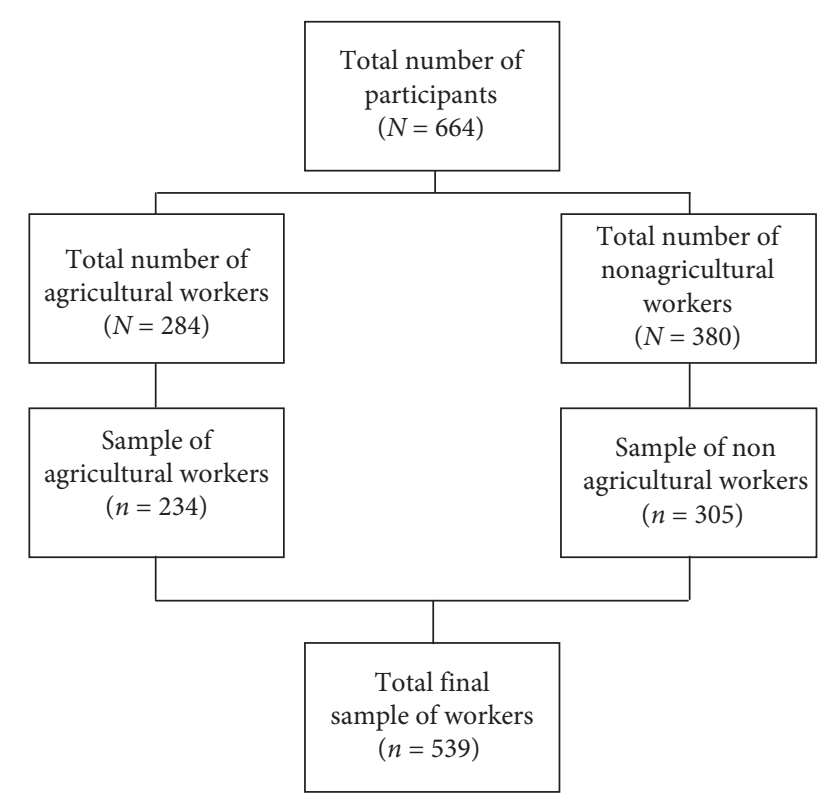

FIgURE 1: Study participants and sample of agricultural and nonagricultural workers.

measure for the early detection of cognitive impairment in the occupational context.

\section{Materials and Methods}

2.1. Participants. A sample of 664 workers participated in this study between 2017 and 2018. Of these, 284 were agricultural workers from the Maule region, and 380 were nonagricultural workers from the Metropolitan and Valparaiso regions (see Figure 1). For the reliability analysis, a subsample of 193 agricultural workers and 193 nonagricultural workers (total $N=386$ ) had been administered both the test and retest (72\% of the total sample). Each participant has signed an informed consent document prior to the beginning of the study. The research protocol and the informed consent documents have been revised and approved by the certified Ethics Committee of the Safety Mutual CChC.

Workers of the exposed group $(n=284)$ were recruited from agricultural companies of the Maule region that accepted to participate in the study, and they were randomly chosen from those who met the inclusion criteria. Inclusion criteria were that participants must be permanent agricultural workers, older than 18 years old, with at least 2 years of occupational exposure to pesticides, and should have normal healthy condition at the moment of the study. Exclusion criteria, on the other hand, were that workers were temporary, younger than 18 years old, and had any health condition (metabolic diseases, or any which could alter the interpretation of results). All this information has been collected through an initial "Pesticides Exposure Questionnaire" already validated in Chile from a previous study [22].

Workers of the control group $(n=380)$ were randomly selected from building companies of other regions (Santiago and Viña del Mar) far from agricultural fields and crops. Inclusion criteria were that they should not be agricultural workers, older than 18, that had not worked (neither temporally) in agriculture for the last 5 years, and that have not been occupationally exposed to any neurotoxic. This information has also been collected by a previous questionnaire.

After a first cleaning process, filtering all the missing data and eliminating subjects that did not meet the inclusion criteria, the sample reached a number of $N=539$ for the analysis (see Figure 1).

\subsection{Materials}

2.2.1. Tests Selection. With the aim to select the most appropriate tests for the brief assessment tool, the content validity has been assessed through an accurate literature review and construct identification. We found different tests of processing speed, attention, and visual memory. In a second phase, an evaluation of the tools by three expert referees' with an average work experience in the field of neuropsychological evaluation of 14 years has been carried out. Based on the results of these two phases, the clockdrawing test (CDT), the frontal assessment battery (FAB), and the trail making tests (TMT) A and B have been chosen to be included in the proposed brief assessment tool. Each of these tests is described in the following sections, and a summary of the functions evaluated by them is presented in Table 1.

2.2.2. Clock-Drawing Test (CDT). The clock-drawing test is a simple paper-and-pencil task which takes a short time to be administered, corrected, and scored. Basic instruction given to the participant is to draw an analog clock displaying the $11: 10$. For the scoring, 20 items are considered, giving 0 or 1 points depending on the accomplishment of what is indicated for each item. The maximum total score is 20 . Based on the study from Mendez and colleagues [24], the binary items could be grouped in aggregated scores. The authors established three groups of items by correlating the scores with other neuropsychological tests. The first group has been strongly associated with visuoperceptual measures. The second group has been associated with attention measures. The third group has not been associated with any correlated test in the aforementioned study and has been considered with items measuring numerical sequencing. In our study, we attained to this grouping, producing three subscores: visuospatial skills (CDT-VS), attention (CDT-ATT), and numerical sequencing (CDT-NS), plus an aggregated total score based on the 20 total score. Three or more errors have been considered as cognitive impairment, and normal subjects show 2 errors or below.

2.2.3. Frontal Assessment Battery (FAB). The frontal assessment battery is a brief test taking no more than 10 minutes to be administered. It is composed of 6 items exploring different abilities (see Table 1) associated to the 
TABLE 1: Neuropsychological tests and their evaluated functions.

\begin{tabular}{lc}
\hline Test & Measured functions \\
\hline Clock-drawing test (CDT; & Visuoperceptual skills \\
Mendez et al. [24]) & Self-monitoring \\
& $\begin{array}{c}\text { Numeric sequencing } \\
\text { Solor execution } \\
\text { Selective attention }\end{array}$ \\
\hline Frontal assessment battery & Abstract reasoning \\
(FAB; Dubois et al.[25]) & Lexical fluidity and mental \\
& flexibility \\
& Self-regulation and interference \\
& resistance \\
Trail making test (TMT-A and & Inhibitory control \\
TMT-B; Partington and Leiter & Environmental autonomy \\
[19]) & Visuospatial skills \\
& Processing speed \\
& Attention and executive \\
& functions (cognitive flexibility) \\
\hline
\end{tabular}

frontal lobe functions, allowing the identification of executive dysfunctions, and the early detection of diseases such as Alzheimer's, Parkinson's and progressive supranuclear paralysis (PSP). Each item can receive a score from 0 to 3 points. Authors only give a general interpretation of the tool, which is relative to the global score of FAB test, allowing evaluating the severity of the executive dysfunction and obtaining a descriptive pattern of the worse area according to the specific items showing a worse performance.

2.2.4. Trail Making Test (TMT). The trail making test is composed of parts A and B. TMT-A requires the subjects to draw continuous lines connecting 25 scrambled numbered circles following the numeric sequential order (from 1 to 25 ). Similarly, TMT-B requires the participant to draw a continuous line connecting alternatively the circles with numbers and those with letters following each its own sequence (i.e., 1, A, 2, B, 3, C, etc.). In both versions, the examiner instructs the subjects to never lift the pen from the paper, and if he/she makes an error, he/she has to return to the circle where the error originated and continue. The final score is represented by the total time (seconds) needed to complete the task. Accordingly, a lower score in TMT expresses a better performance, while longer times mean that the subject had problems completing the task. As a cutoff for abnormal performance, commonly 78 or more seconds for TMT-A and 273 or more seconds for TMT-B for completion time are considered.

Besides the tools described above, the Chilean-standardized Spanish version of the Wechsler Adults Intelligence Scale (WAIS-IV [23]) has been administered to the $18 \%$ of the agricultural workers sample $(n=41)$ as a gold standard to compare performances obtained with the brief assessment tool. The administration of this test took between 1 hour and half and 2 hours for each worker.

2.3. Procedures. Assistants received an intensive training in the Laboratory of the Neuropsychology and Cognitive
Neurosciences Research Center (CINPSI Neurocog) in the Catholic University of Maule (UCM, Talca, Chile) previously to the beginning of all the test administrations.

Before starting the administration of the brief assessment tool, a preliminary pilot assessment including the whole set of tests has been given to 6 agricultural workers in order to fix any problems with the tool's performance. From the analysis of the performances of this preliminary sample, it was verified that the tests worked fine and did not need any change or modification to the compiled administration protocol.

The final assessment protocol has been administered to test takers in two times, with an intermediate lapse of 2-4 weeks. All tests have been given in their workplaces, and each company facilitated an appropriate space (without too much noise or interferences) for test administration. Each assessment was performed by an assistant to a worker at a time. The test taker had to read the informed consent and eventually sign a participation agreement. Then, the assistant explained briefly the sequence of actions he/she was going to perform and answered his/her eventual questions.

2.4. Analyses. Statistical analyses have been performed with R-Studio [26] and SPSS 24.0 [27]. Before starting the analyses, a coresearcher and a research assistant checked 100\% of the protocols for recording integrity and correctness of scoring. Firstly, an extensive exploratory analysis has been performed to individuate all the missing data in the examined variables. After that, the variables have been checked for normal distribution with the Shapiro-Wilk test. On the basis of the results of this analysis (all the tests had a $p$ value less than 0.001), nonparametric statistical tests were performed for the following analysis of data. Nonparametric correlations (Spearman's rho) have been performed on the test-retest evaluation, while Cronbach's alpha coefficient has been calculated to measure internal consistency of the brief assessment tool. For the construct validity test, a factor analysis has been performed, by means of which the eigenvalues $>1$ have been extracted with a maximum of 25 iterations to obtain convergence. Also, a direct oblimin rotation method has been applied to generate the rotated factor solution, considering the same 25 iterations to converge. The Mann-Whitney $U$ test has been performed for the means comparisons between the two groups. An analysis of the convergent validity was made with a subsample of 41 workers to whom the WAIS-IV test was administered as a Gold Standard measure. This test is an intelligence scale composed of 10 subscales, which yields a total score that after being transformed to the standard score represents the intellectual quotient (IQ) of the test takers. In addition to the above, the estimation of the normative values of the brief scale by age group and educational level was made from the percentiles represented by each stratified score.

\section{Results and Discussion}

3.1. Descriptive Analysis. For this analysis, 539 workers have been considered (agricultural, $n=234$; nonagricultural, $n=305)$. From the agricultural workers sample, $28 \%$ of them 
TABLE 2: Demographic characteristics by group.

\begin{tabular}{lcc}
\hline & & Group \\
& Agricultural $(n=234)$ & Nonagricultural $(n=305)$ \\
\hline Mean age (DS) & $46.26(12.04)$ & $41.13(13.40)$ \\
Female percentage & $20.5 \%$ & $6.2 \%$ \\
Mean monthly income & $623 \mathrm{USD}$ & 1044 USD \\
\hline Educational level & & \\
Illiterate & $0.9 \%$ & $1.0 \%$ \\
Primary (incomplete) & $25.2 \%$ & $10.8 \%$ \\
Primary (complete) & $34.6 \%$ & $16.4 \%$ \\
Secondary (incomplete) & $12.4 \%$ & $21.3 \%$ \\
Secondary (complete) & $26.9 \%$ & $44.3 \%$ \\
Technical/professional & $0.0 \%$ & $6.2 \%$ \\
\hline
\end{tabular}

TABLE 3: Tests scores by group, including mean, standard deviation, median, minimum, and maximum.

\begin{tabular}{|c|c|c|c|c|c|c|c|c|c|c|c|c|}
\hline & \multicolumn{12}{|c|}{ Group } \\
\hline & \multicolumn{6}{|c|}{ Agricultural $(n=234)$} & \multicolumn{6}{|c|}{ Nonagricultural $(n=305)$} \\
\hline & Obs. & M & DS & Med & Min & Max & Obs. & M & DS & Med & Min & Max \\
\hline CDT-VS & 234 & 9.49 & 1.724 & 10 & 0 & 11 & 305 & 9.95 & 1.494 & 10 & 1 & 11 \\
\hline CDT-ATT & 234 & 3.53 & 0.759 & 4 & 0 & 4 & 305 & 3.77 & 0.584 & 4 & 0 & 4 \\
\hline CDT-NS & 234 & 4.47 & 1.187 & 5 & 0 & 5 & 305 & 4.73 & 0.823 & 5 & 0 & 5 \\
\hline CDT total & 234 & 17.49 & 3.175 & 19 & 1 & 20 & 305 & 18.45 & 2.536 & 19 & 1 & 20 \\
\hline FAB1 & 234 & 1.93 & 0.893 & 2 & 0 & 3 & 304 & 2.15 & 0.831 & 2 & 0 & 3 \\
\hline FAB2 & 234 & 1.96 & 0.978 & 2 & 0 & 3 & 304 & 2.19 & 0.853 & 2 & 0 & 3 \\
\hline FAB3 & 234 & 2.48 & 0.819 & 3 & 0 & 3 & 304 & 2.67 & 0.652 & 3 & 0 & 3 \\
\hline FAB4 & 234 & 2.56 & 0.780 & 3 & 0 & 3 & 304 & 2.70 & 0.648 & 3 & 0 & 3 \\
\hline FAB5 & 234 & 2.26 & 0.999 & 3 & 0 & 3 & 304 & 2.47 & 0.915 & 3 & 0 & 3 \\
\hline FAB6 & 234 & 2.95 & 0.282 & 3 & 0 & 3 & 304 & 2.97 & 0.256 & 3 & 0 & 3 \\
\hline FAB total & 234 & 14.13 & 2.894 & 15 & 5 & 18 & 304 & 15.17 & 2.462 & 16 & 5 & 18 \\
\hline TMT-A & 234 & 63.38 & 34.412 & 56 & 11 & 285 & 304 & 54.13 & 29.864 & 48 & 16 & 301 \\
\hline TMT-B & 193 & 141.1 & 63.634 & 128 & 31 & 401 & 277 & 122.63 & 67.953 & 103 & 32 & 301 \\
\hline
\end{tabular}

currently apply pesticides and $37.2 \%$ made the last application during 2 years or less. A $28 \%$ of this sample applies pesticides only as a temporary job, while $13.7 \%$ does it permanently. A $27 \%$ applied pesticides since less than 10 years, while $21.4 \%$ applied since 10 years or above. A $33.3 \%$ declares to have a certification of pesticide applicator. A $57.7 \%$ declare to be aware of the health hazards of the application of pesticides, and $50.4 \%$ stated that they have received training on these hazards. A $42.3 \%$ declare wearing personal protective equipment (PPE) during their work when they mixed the pesticides, and a $44 \%$ said that they changed their clothes after apply chemicals at work. Only $7.7 \%$ referred that they have been intoxicated and 3.4\% that they were hospitalized after pesticides poisoning. With regard to symptoms, a $15 \%$ have experienced dizziness, nausea, fatigue, vomiting, and/or salivation while applying pesticides. A $38.5 \%$ had a cholinesterase test, and only $3.8 \%$ had an exam that indicated severe poisoning. Demographic characteristics of each group composition are shown in Table 2.

Also, descriptive statistics about the scores obtained for each group in each administered test have been calculated (see Table 3).

3.2. Content Validity. As described above, construct domains have been qualitatively identified during the tool development phase through an extensive literature review that allowed to find tests that were brief, were of public domain, and addressed the assessment of the cognitive functions of importance related to the neurotoxic effects of the most used pesticides in Chile. Then, they were revised by a pool of experts on the field of neuropsychology to evaluate their content validity. The expert judges totally agreed that CDT measured visuospatial skills (100\% agreement), while partially agreed that this test measures focused attention, planning and organizational abilities, and inhibitory control. With respect to the FAB, they completely agreed (100\%) that it measures planning and inhibitory control and partially agreed $(75 \%)$ that it measures working memory, sustained attention, and organization ability. Regarding TMT-A, there was total agreement (100\%) that it measures processing speed and partial agreement (75\%) about focused and selective attention, planning, inhibitory control, and visuospatial skills. Finally, in the case of TMT-B, judges agreed $(100 \%)$ that it consists in a working memory and sustained attention measurement and partially agreed (75\%) that it is a self-monitoring measurement. From these interjudge evaluations, the test of the brief assessment tool with strongest relative weight was the $\mathrm{FAB}$, and progressively lower weights resulted for TMT-A, CDT, and TMT-B. The cognitive function of simultaneous processing did not reach agreement for any evaluated test and therefore is not considered 


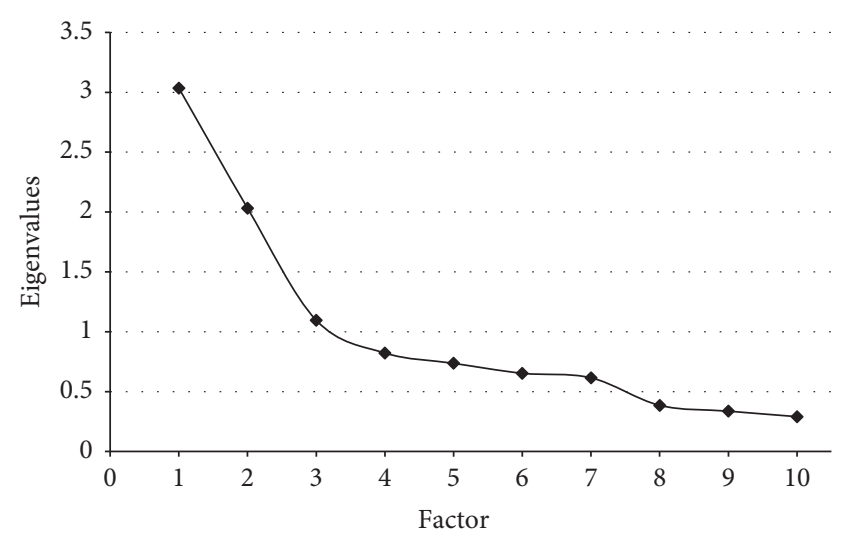

FIgURE 2: Screen plot with the eigenvalues of each factor yielded from the factor analysis.

as a construct included in the brief assessment tool, while focused attention and self-monitoring reached the lowest interjudge agreement level. On the other hand, the highest agreement values have been reached by visuospatial skills, selective attention, planning, processing speed, and inhibitory control.

3.3. Internal Consistency. Internal consistency analyses have been performed calculating Cronbach's alpha for each subtest, except TMT-A and TMT-B, since their scores represented times of completion and were not comparable. CDT has an alpha $=0.838$, indicating a high internal consistency for its 20 items. FAB's initial analysis on all the 6 items had an alpha $=0.606$; eliminating the item 6 , internal consistency reached alpha $=0.633$, considered as satisfactory value. For this reason, in the following analyses, this item has been not considered.

3.4. Construct Validity. Construct validity has been calculated with factor analyses on scores of the entire sample $(N=539)$. A number of 10 variables have been considered as input variables, including the $3 \mathrm{CDT}$ aggregated items (visuospatial, attention, and numerical sequencing), 5 FAB items, and TMT-A and TMT-B scores. The sample obtained a $\mathrm{KMO}=0.742$ at the Kaiser-Meyer-Olkin value (recommended value is 0.6). The Bartlett's sphericity test was applied and resulted in a value of $\chi^{2}(45)=1291, p<0.001$, which indicates that the correlation structure is adequate to perform factor analyzes. The principal axis factoring (PAF) was used as the extraction method, since the data of the tools were not normally distributed [28]. Then, the instrument was subjected to a direct oblimin rotation method with a cutoff of 0.30 and using the Kaiser's criterion of eigenvalues greater than 1, which yielded a three-factor solution as the best fit for the data, accounting for $61.62 \%$ of the variance. The factors four to ten resulted with eigenvalues below 1 (Figure 2), only explaining between $8 \%$ and $2 \%$ of variance. The results of this factor analysis are summarized in Table 4. The item 2 of the FAB was eliminated, since it was not integrated into any of the factor solutions tested, with factor weight less than 0.30 .
The representation of the resulting three-factor solution was made using the labels that emerged from the evaluation of expert judges with respect to the skills measured by the tests included in the brief assessment tool. After the interpretation of the components as described, the following descriptors were obtained for each one: (a) visuospatial skills and processing speed: this factor had an eigenvalue of 3.03 and accounted for $30.34 \%$ of the variance; (b) planning: the eigenvalue of this factor was 2.03 and accounted for $20.31 \%$ of the variance; (c) selective attention and inhibitory control: this factor had an eigenvalue of 1.09 and accounted for $10.9 \%$ of the variance.

We compared means of each test between groups with the nonparametric Mann-Whitney $U$ test, observing that each scale has significant different scores in both groups $(p<0.001)$. Results and values are shown in detail in Table 5.

3.5. Convergent Validity. To examine convergent validity of the factor solution, the WAIS-IV test was used as a gold standard, and it was administered to $18 \%$ of the sample of agricultural workers $(N=41)$. From this sample, it is verified that the instruments have a significant association with the performance assessed by the gold standard test. First off, all the tests of the brief assessment tool showed a significant correlation with the full-scale IQ score. The tools that correlate with all the four factor indexes of the WAIS-IV are the adjusted FAB (FABadj) and the TMT-A. The CDT correlates only with the perceptual reasoning index, and the TMT-B correlates with the perceptual reasoning index as well, but also with the working memory index. The results of the convergent validity analysis are detailed in Table 6.

3.6. Test-Retest Reliability. For the estimation of the brief assessment tool's reliability in terms of its stability in time, a subsample of participants from both groups has been given the brief protocol twice, with an interval period after the first administration of about 2-4 weeks. The correlation between both periods was estimated with Spearman's rho test for each assessment tool, resulting in all the values to be significantly correlated and, hence, stable in time. Detailed values for each test and group are shown in Table 7 .

3.7. Normative Data of the Brief Assessment Tool Stratified for Age and Educational Level. Correlations among the demographical variables (age, educational level, and household monthly income) and scores on each of the tests show that only age and educational level were correlated with the scores of all the instruments of the brief assessment tool (see Table 8). Therefore, stratification of the normative data according to these 2 variables has been performed.

In the case of the variable age, it was divided into four groups based on the age distribution of the sample, taking into account the quartiles observed. On the other hand, for the educational level, it was stratified into two groups, the first from 0 to 8 years of education, composed by workers with an educational attainment of elementary educational level, complete or incomplete. The second group is 
TABLE 4: Exploratory factor analysis results for the tests of the brief scale.

\begin{tabular}{|c|c|c|c|c|}
\hline \multirow{2}{*}{ Items } & \multicolumn{3}{|c|}{ Factor* } & \multirow{2}{*}{ Dimension } \\
\hline & 1 & 2 & 3 & \\
\hline $\begin{array}{l}\text { TMT-A } \\
\text { TMT-B }\end{array}$ & $\begin{array}{l}-0.855 \\
-0.803\end{array}$ & & & Visuospatial skills and processing speed \\
\hline $\begin{array}{l}\text { CDT-VS } \\
\text { CDT-ATT } \\
\text { CDT-NS } \\
\end{array}$ & & $\begin{array}{l}0.761 \\
0.814 \\
0.791 \\
\end{array}$ & & Planning \\
\hline $\begin{array}{l}\text { FAB1 } \\
\text { FAB2 } \\
\text { FAB3 } \\
\text { FAB4 } \\
\text { FAB5 }\end{array}$ & - & - & $\begin{array}{c}0.433 \\
- \\
0.718 \\
0.505 \\
0.494\end{array}$ & Selective attention and inhibitory control \\
\hline
\end{tabular}

FAB items description: FAB1: similarities; FAB2: lexical fluidity and flexibility; FAB3: sequences (programming); FAB4: conflicting instructions; FAB5: go/no go. * Only values of factor weight higher than 0.30 are shown.

TABle 5: Group means comparison of performance in each test of the brief assessment tool included in the factor solution.

\begin{tabular}{|c|c|c|c|c|c|}
\hline \multirow{2}{*}{ Test } & \multicolumn{2}{|c|}{ Group } & \multirow{2}{*}{$U$} & \multirow{2}{*}{$z$} & \multirow{2}{*}{$p$} \\
\hline & Agricultural (exposed) & Nonagricultural (not exposed) & & & \\
\hline CDT-VS & 239.26 & 293.58 & 28492.5 & -4.21 & 0.000 \\
\hline CDT-ATT & 242.57 & 290.23 & 29266 & -4.70 & 0.000 \\
\hline CDT-NS & 256.49 & 279.51 & 32523.5 & -2.60 & 0.000 \\
\hline FABadj* $^{*}$ & 240.8 & 291.59 & 28851.5 & -3.82 & 0.000 \\
\hline TMT-A & 302.04 & 244.45 & 27953 & -4.26 & 0.000 \\
\hline TMT-B & 267.27 & 213.36 & 20598.5 & -4.23 & 0.000 \\
\hline
\end{tabular}

FABadj includes only items 1,3 , 4, and 5 from the original FAB.

TABle 6: Correlations coefficients (Spearman's rho) of each test included in the brief assessment tool and WAIS' FSIQ and indexes.

\begin{tabular}{|c|c|c|c|c|c|c|c|c|}
\hline & FSIQ & VCI & PRI & WMI & PSI & CDT & FABadj & TMT-A \\
\hline$\overline{\text { FSIQ }}$ & 1 & & & & & & & \\
\hline ICV & $0.72^{* *}$ & 1 & & & & & & \\
\hline IRP & $0.76^{* *}$ & 0.28 & 1 & & & & & \\
\hline IMT & $0.78^{* *}$ & $0.61^{* *}$ & $0.55^{*}$ & 1 & & & & \\
\hline IVP & $0.63^{* *}$ & $0.34^{*}$ & $0.36^{*}$ & 0.29 & 1 & & & \\
\hline CDT & $0.3^{*}$ & 0.42 & $0.39^{*}$ & 0.20 & 0.31 & 1 & & \\
\hline FAB Adj & $0.47^{* *}$ & $0.32^{*}$ & $0.31^{*}$ & $0.49^{*}$ & $0.33^{*}$ & $0.21^{*}$ & 1 & \\
\hline TMT-A & $-0.57^{* *}$ & $-0.4^{*}$ & -0.51 & $-0.32^{*}$ & $-0.49^{*}$ & $-0.29^{* *}$ & $-0.45^{* *}$ & 1 \\
\hline TMT-B & $-0.41^{*}$ & -0.3 & $-0.36^{*}$ & $-0.39^{*}$ & -0.2 & $-0.25^{* *}$ & $-0.49^{* *}$ & $-0.7^{* *}$ \\
\hline
\end{tabular}

WAIS-IV indexes: FSIQ total = full-scale intelligent quotient; $\mathrm{VCI}=$ verbal comprehension index; $\mathrm{PRI}=$ perceptual reasoning index; $\mathrm{WMI}=$ working memory index; PSI $=$ processing speed index. ${ }^{*}=p<0.05 ;{ }^{* *}=p<0.005$.

composed of participants who had between 8 and 12 years of studies, having an incomplete or complete high school educational attainment.

Figures 3 and 4 show the mean scores of the tests included in the brief assessment tool for each age group and according to the educational level, based on the stratification previously described. It is evident that in all the tests there is a detriment in the performance at older age, being more pronounced in the case of the group with lower educational level.

Table 9 shows the normative data of Chilean agricultural workers for the brief validated assessment tool, transformed into percentiles for each age group and educational level. It should be noted that for the older age group, there were no subjects with more than eight years of education. Therefore, for that group are only assumed for people with eight or less years of education.

\section{Discussion}

We have presented the process and the main results of the validation and reliability study of a brief screening scale to measure cognitive deterioration in occupational populations that are exposed to neurotoxins through the widespread use of pesticides. To date, there have been no similar studies with tools administered in Chile with these characteristics and that allow knowing in a specific way how they work with agricultural workers to monitor health and any signs of cognitive deterioration.

The main purpose has been achieved in terms of developing a tool composed of public domain tests, with no cost, simple to apply, and that could be administered by health and safety technicians due to its ease of administration and punctuation. The abridged tool complies with all 
TABLE 7: Reliability (stability) of test-retest scores in both groups.

\begin{tabular}{lccc}
\hline Test and groups & Obs. & Rho & $p$ \\
\hline Agricultural & & & \\
CDT-total & 196 & 0.477 & 0.000 \\
CDT-VS & 196 & 0.475 & 0.000 \\
CDT-ATT & 196 & 0.408 & 0.000 \\
CDT-NS & 196 & 0.421 & 0.000 \\
FABadj & 196 & 0.659 & 0.000 \\
TMT-A & 194 & 0.733 & 0.000 \\
TMT-B & 194 & & \\
\hline Nonagricultural & & 0.553 & 0.000 \\
CDT-total & 193 & 0.549 & 0.000 \\
CDT-VS & 193 & 0.445 & 0.000 \\
CDT-ATT & 193 & 0.440 & 0.000 \\
CDT-NS & 193 & 0.384 & 0.000 \\
FABadj & 193 & 0.726 & 0.000 \\
TMT-A & 193 & 0.660 & 0.000 \\
TMT-B & 177 & & \\
\hline
\end{tabular}

TABLE 8: Correlations among age, education, and household income with the tests.

\begin{tabular}{|c|c|c|c|c|c|c|}
\hline & Age & Education & Family income & CDT & FABadj & TMT-A \\
\hline Age & 1 & & & & & \\
\hline Education & $-0.60^{* *}$ & 1 & & & & \\
\hline Household income & -0.06 & 0.06 & 1 & & & \\
\hline CDT & $-0.16^{*}$ & $0.22^{* *}$ & 0.04 & 1 & & \\
\hline FABadj & $-0.35^{* *}$ & $0.37^{* *}$ & 0.06 & 0.12 & 1 & \\
\hline TMT-A & $0.51^{* *}$ & $-0.40^{* *}$ & -0.08 & $-0.24^{* *}$ & $-0.39^{* *}$ & 1 \\
\hline TMT-B & $0.45^{* *}$ & $-0.39^{* *}$ & -0.08 & $-0.21^{* *}$ & $0.46^{* *}$ & $-0.65^{* *}$ \\
\hline
\end{tabular}

${ }^{*} p<0.05 ;{ }^{* *} p<0.005$.
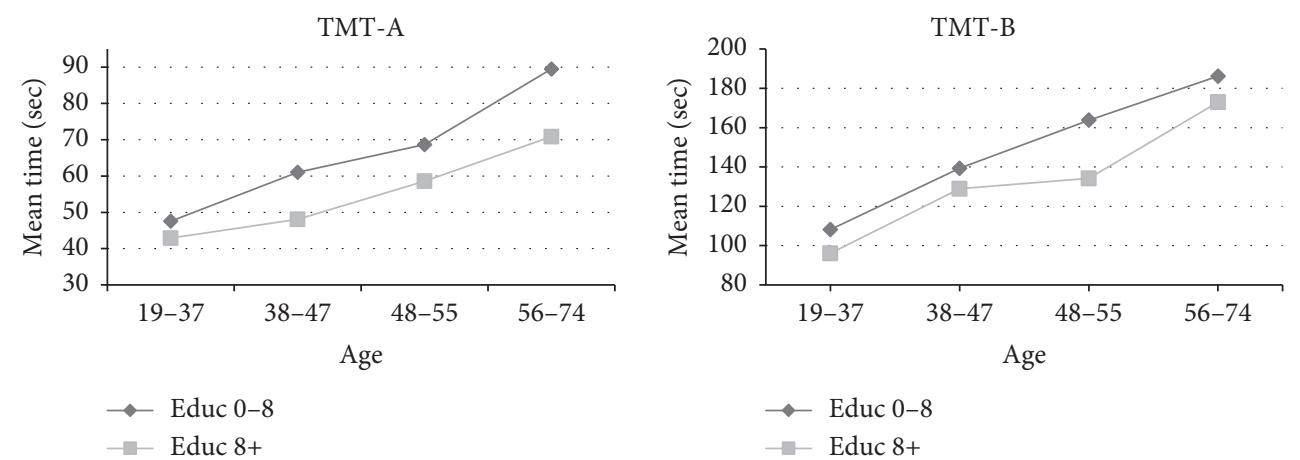

Figure 3: Performance in TMT-A and TMT-B, according to the four age groups and the two educational levels.

the above characteristics and has also been proven to have content and construct validity with respect to the measurement of skills that are associated with those that according to the literature are among the most deteriorated in the face of chronic exposure to acetylcholinesterase inhibitory pesticides, which are the most commonly sold and used pesticides in the Chilean agricultural setting. Previous studies have shown that OP exposure impacts neurological wellbeing such as the executive functions, such as attention (sustained and focused), planning, inhibitory control, and also the visuospatial ability and processing speed [3-7]. Based on the criterion of judges, the content validity was positively verified, since the brief tests included addressed the measurement of these skills and then their pertinence was verified from the underlying factor structure with the three factors that group the different parts of the scale: (1) visuospatial skill and processing speed; (2) planning capacity; (3) selective attention and inhibitory control.

For the study, we administered the gold standard measure (WAIS-IV) to compare the scores obtained from the scale tests and evaluate their convergent validity. Our results showed that in the agricultural occupational population, the TMT-A and the FABadj are the measures most correlated with the level of global intellectual functioning (FSIQ). It is worth mentioning that all the tests included in the brief assessment tool correlate with the FSIQ or with 

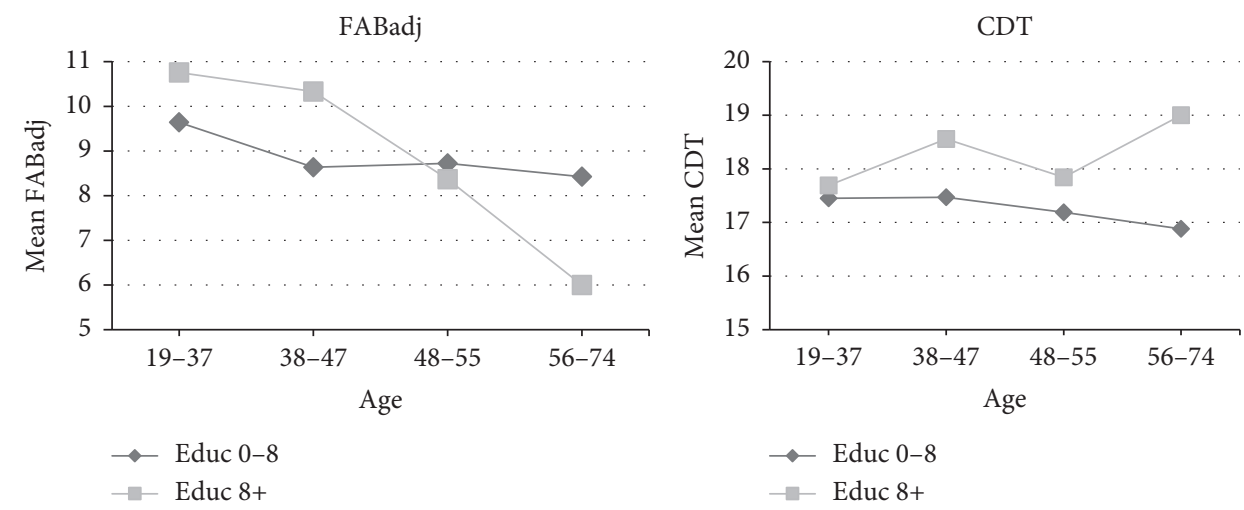

Figure 4: Performance in FABadj and CDT, for the four age groups and the 2 educational levels.

TABle 9: Percentiles for the brief assessment tool for each normative group.

\begin{tabular}{|c|c|c|c|c|c|c|c|c|}
\hline \multirow{2}{*}{ Percentile } & \multicolumn{4}{|c|}{ Education $0-8$ years } & \multicolumn{4}{|c|}{ Education $8+$ years } \\
\hline & $\mathrm{CDT}$ & FABadj & TMT-A & TMT-B & $\mathrm{CDT}$ & FABadj & TMT-A & TMT-B \\
\hline \multicolumn{9}{|c|}{$19-37$ years old $(n=56)$} \\
\hline 95 & 20 & 12 & 33 & 72 & 20 & 12 & 22 & 53 \\
\hline 90 & 20 & 12 & 33 & 72 & 20 & 12 & 26 & 62 \\
\hline 75 & 19 & 12 & 34 & 75 & 20 & 12 & 32 & 73 \\
\hline 50 & 19 & 10 & 39 & 94 & 19 & 11 & 50 & 102 \\
\hline 25 & 17 & 8 & 52 & 124 & 16 & 10 & 58 & 128 \\
\hline 10 & 10 & 6 & 67 & 143 & 12 & 9 & 63 & 177 \\
\hline 5 & 9 & 6 & 67 & 143 & 9 & 8 & 69 & 195 \\
\hline \multicolumn{9}{|c|}{$38-47$ years old $(n=63)$} \\
\hline 95 & 20 & 12 & 30 & 81 & 20 & 12 & 27 & 54 \\
\hline 90 & 20 & 12 & 33 & 90 & 20 & 12 & 30 & 59 \\
\hline 75 & 19 & 11 & 43 & 109 & 20 & 12 & 37 & 81 \\
\hline 50 & 18 & 9 & 55 & 135 & 19 & 11 & 45 & 121 \\
\hline 25 & 18 & 7 & 69 & 183 & 18 & 9 & 57 & 146 \\
\hline 10 & 12 & 3 & 87 & 248 & 14 & 7 & 80 & 216 \\
\hline 5 & 6 & 2 & 107 & 294 & 13 & 5 & 87 & 318 \\
\hline \multicolumn{9}{|c|}{$48-55$ years old $(n=62)$} \\
\hline 95 & 20 & 11 & 39 & 65 & 20 & 11 & 33 & 73 \\
\hline 90 & 20 & 11 & 42 & 93 & 20 & 11 & 37 & 79 \\
\hline 75 & 19 & 11 & 49 & 114 & 20 & 11 & 48 & 107 \\
\hline 50 & 18 & 9 & 62 & 149 & 19 & 8 & 58 & 129 \\
\hline 25 & 16 & 8 & 79 & 199 & 17 & 6 & 64 & 168 \\
\hline 10 & 13 & 5 & 93 & 251 & 14 & 5 & 85 & 194 \\
\hline 5 & 10 & 2 & 109 & 274 & 12 & 4 & 85 & 194 \\
\hline \multicolumn{9}{|c|}{$56-74$ years old $(n=53)$} \\
\hline 95 & 20 & 12 & 36 & 95 & - & - & - & - \\
\hline 90 & 20 & 11 & 45 & 104 & - & - & - & - \\
\hline 75 & 19 & 10 & 58 & 116 & - & - & - & - \\
\hline 50 & 18 & 9 & 79 & 168 & - & - & - & - \\
\hline 25 & 16 & 7 & 104 & 220 & - & - & - & - \\
\hline 10 & 12 & 3 & 143 & 311 & - & - & - & - \\
\hline 5 & 7 & 3 & 197 & 370 & - & - & - & - \\
\hline
\end{tabular}

some index of cognitive functioning measured by the Gold Standard test.

The reliability was verified both in terms of internal consistency of the tests, with values from satisfactory to high consistency, and in terms of stability over time of the measurement through test and retest. Even though all the tests of the scale showed a high and significant stability in its administration over time, the Trail Making test stands out especially in this sense, being the test with greater stability as a measure in the agricultural population.

It is expected that in the case of the CDT, the scores obtained will be from 15 points up, with a low difference expected between those cases that show deterioration compared to those who do not. In the case of the FAB, the psychometric analysis itself has led to a decrease in the number of items, because two of its original items (2 and 6) 
had little association with the factor solution. To administer this brief assessment tool, the items are maintained in its original composition for the CDT test and for the TMT-A and TMT-B tests. To administer the FAB, a most brief version named "FABadj" here should be used, which considers only 4 of the 6 original items $(1,3,4$, and 5$)$. For the interpretation of the test from the norms reported here, it is advisable to follow the sequence given below:

(1) Set the age group and education level of the evaluated person

(2) Transform the raw score to percentile according to the normative group of the test taker

(3) Report the percentiles obtained for each of the 3 factors that would be measured by each test of the scale: Visuoperceptual ability and processing speed (TMT-A, TMT-B), planning capacity (CDT), and selective attention and inhibitory control (FABadj)

A percentile of 25 or below indicates a cognitive deterioration in the specific factor measured. It should be considered that in the last normative group, the oldest, there were no subjects in the sample with more than eight years of study, so when the scale is administered to people over 56 years old, the data of the only age group available should be used to convert to percentiles. It should also be mentioned that although the TMT-B test is part of the validated scale, in our case, we observed that there were around $15 \%$ of cases where it was not possible to complete the administration because it requires knowledge of the sequence of the alphabet. It is expected that when the TMT$\mathrm{B}$ is administered in an agricultural population, this type of situation could be found in workers with a very low educational level. In those cases, it would be convenient to suspend the administration of this part of the brief assessment tool in particular. Also, in cases of the TMT-B, when the test taker has a completion time of more than 300 seconds, it is advisable to suspend their administration because they have exceeded what is conventionally stated as a time limit. This would also be advisable considering the normative data obtained in the present study for agricultural occupational population since there were a minimum percentage of cases (around 1\%) that had longer times of completion at that level. Therefore, it is advisable to suspend the administration of TMT-B, because in any case, its performance would be located within the $5 \%$ of worst performance according to the norm.

\section{Conclusion}

It can be concluded that the aim of this work has been met satisfactorily, establishing the validity and reliability of a brief assessment tool for the monitoring of health effects due to permanent occupational exposure to neurotoxic pesticides. Also, for the first time in Chile, there are specific norms for agricultural workers population, with instructions for administration and interpretation with ease for its potential extended use in the prevention of cognitive deterioration in the health of agricultural workers.

\section{Data Availability}

The datasets are available from the first corresponding author on reasonable request. The ethical approval granted to the authors does not allow the publication of the dataset online. If readers would like to reanalyse the dataset, additional ethical approvals (on an individual user and purpose basis) will be required.

\section{Disclosure}

This work was selected in the Call for Projects of Research and Innovation in Prevention of Accidents and Professional Illnesses 2016 of the Superintendence of Social Security (Superintendencia de Seguridad Social del Gobierno de Chile, SUSESO).

\section{Conflicts of Interest}

The authors declare that there are no conflicts of interest regarding the publication of this paper.

\section{Acknowledgments}

The authors would like to thank Verónica Miranda who provided great help during the research. Also, the authors would like to thank all the research assistants that provided help during the research, including undergraduate students of the Department of Nursery and the Department of Psychology. This work was financed by Mutual de Seguridad CChC with funds of the Law of Social Security (No. 16.744) on Accidents at Work and Occupational Diseases.

\section{References}

[1] D. Tshala-Katumbay, J.-C. Mwanza, D. S. Rohlman, G. Maestre, and R. B. Oriá, "A global perspective on the influence of environmental exposures on the nervous system," Nature, vol. 527, no. 7578, pp. S187-S192, 2015.

[2] A. Kolk, "The social responsibility of international business: from ethics and the environment to CSR and sustainable development," Journal of World Business, vol. 51, no. 1, pp. 23-34, 2016.

[3] M. Saunders, B. L. Magnanti, S. Correia Carreira et al., "Chlorpyrifos and neurodevelopmental effects: a literature review and expert elicitation on research and policy," Environmental Health, vol. 11, no. 1, p. S5, 2012.

[4] M. T. Muñoz-Quezada, B. A. Lucero, D. B. Barr et al., "Neurodevelopmental effects in children associated with exposure to organophosphate pesticides: a systematic review," Neurotoxicology, vol. 39, pp. 158-168, 2013.

[5] S. M. Ross, I. C. McManus, V. Harrison, and O. Mason, "Neurobehavioral problems following low-level exposure to organophosphate pesticides: a systematic and meta-analytic review," Critical Reviews in Toxicology, vol. 43, no. 1, pp. 21-44, 2013.

[6] J. N. Lin, C. L. Lin, M. C. Lin et al., "Increased risk of dementia in patients with acute organophosphate and carbamate poisoning: a nationwide population-based cohort study," Medicine, vol. 94, no. 29, p. e1187, 2015.

[7] M. T. Muñoz-Quezada, B. A. Lucero, V. P. Iglesias et al., "Chronic exposure to organophosphate (OP) pesticides and 
neuropsychological functioning in farm workers: a review," International Journal of Occupational and Environmental Health, vol. 22, no. 1, pp. 68-79, 2016.

[8] Food and Agricultural Organization, International Code of Conduct for the Distribution and Use of Pesticides Revised Version, Food and Agricultural Organization, Rome, Italy, 2006.

[9] SAG (Servicio Agrícola y Ganadero), Report on Sales of Pesticides for Agricultural Use in Chile, SAG (Servicio Agrícola y Ganadero), Santiago de Chile, 2012, http://www.sag.gob.cl/ sites/default/files/declaracion_de_venta_de_plaguicidas_ano_ 2012.pdf.

[10] S. Suratman, J. W. Edwards, and K. Babina, "Organophosphate pesticides exposure among farmworkers: pathways and risk of adverse health effects," Reviews on Environmental Health, vol. 30, pp. 65-79, 2015.

[11] D. S. Rohlman, W. K. Anger, and P. J. Lein, "Correlating neurobehavioral performance with biomarkers of organophosphorous pesticide exposure," Neurotoxicology, vol. 32, no. 2, pp. 268-276, 2011.

[12] F. Sánchez-Santed, M. T. Colomina, and E. Herrero Hernández, "Organophosphate pesticide exposure and neurodegeneration,” Cortex, vol. 74, pp. 417-426, 2016.

[13] W. K. Anger, M. G. Cassitto, Y. X. Liang et al., "Comparison of performance from three continents on the WHO-recommended neurobehavioral core test battery," Environmental Research, vol. 62, no. 1, pp. 125-147, 1993.

[14] X. Zhang, M. Wu, H. Yao et al., "Pesticide poisoning and neurobehavioral function among farm workers in Jiangsu, People's Republic of China," Cortex, vol. 74, pp. 396-404, 2016.

[15] WHO, Operational Guide for the WHO Neurobehavioral, World Health Organization, vol. 5, p. 25, Geneva, Switzerland, 1986.

[16] W. Kent Anger, "Neurobehavioural tests and systems to assess neurotoxic exposures in the workplace and community," Occupational and Environmental Medicine, vol. 60, no. 7, pp. 531-538, 2003.

[17] W. K. Anger, Y. X. Liang, V. Nell et al., "Lessons learned--15 years of the WHO-NCTB: a review," Neurotoxicology, vol. 21, no. 5, pp. 837-846, 2000.

[18] W. K. Anger, "Reconsideration of the WHO NCTB strategy and test selection," Neurotoxicology, vol. 45, pp. 224-231, 2014.

[19] J. Partington and R. Leiter, "Partington's pathways test," The Psychological Service Center Journal, vol. 1, pp. 11-20, 1949.

[20] N. M. Gatto, M. Cockburn, J. Bronstein, A. D. Manthripragada, and B. Ritz, "Well-water consumption and Parkinson's disease in rural California," Environmental Health Perspectives, vol. 117, no. 12, pp. 1912-1918, 2009.

[21] K. M. Hayden, M. C. Norton, D. Darcey et al., "Occupational exposure to pesticides increases the risk of incident AD: the cache county study," Neurology, vol. 74, no. 19, pp. 1524-1530, 2010.

[22] M. T. Muñoz-Quezada, B. Lucero, V. Iglesias et al., "Plaguicidas organofosforados y efecto neuropsicológico y motor en la región del Maule, Chile," Gaceta Sanitaria, vol. 30, no. 3, pp. 227-231, 2016.

[23] R. Rosas, M. Tenorio, M. Pizarro et al., "Estandarización de la Escala Wechsler de Inteligencia Para Adultos-Cuarta Edición en Chile," Psykhe, vol. 23, no. 1, pp. 1-18, 2014.

[24] M. F. Mendez, T. Ala, and K. L. Underwood, "Development of scoring criteria for the clock drawing task in Alzheimer's disease," Journal of the American Geriatrics Society, vol. 40, no. 11, pp. 1095-1099, 1992.

[25] B. Dubois, A. Slachevsky, I. Litvan, and B. Pillon, "The FAB: a frontal assessment battery at bedside," Neurology, vol. 55, no. 11, pp. 1621-1626, 2000.

[26] R Core Team, R: A Language and Environment for Statistical Computing, R Foundation for Statistical Computing, Vienna, Austria, 2016.

[27] IBM Corp, IBM SPSS Statistics for Windows, New York, NY, USA, 2016.

[28] A. B. Costello and J. W. Osborne, "Best practices in exploratory factor analysis: four recommendations for getting the most from your analysis," Practical Assessment, Research \& Evaluation, vol. 10, no. 7, pp. 1-9, 2005. 


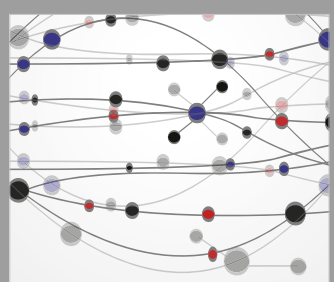

The Scientific World Journal
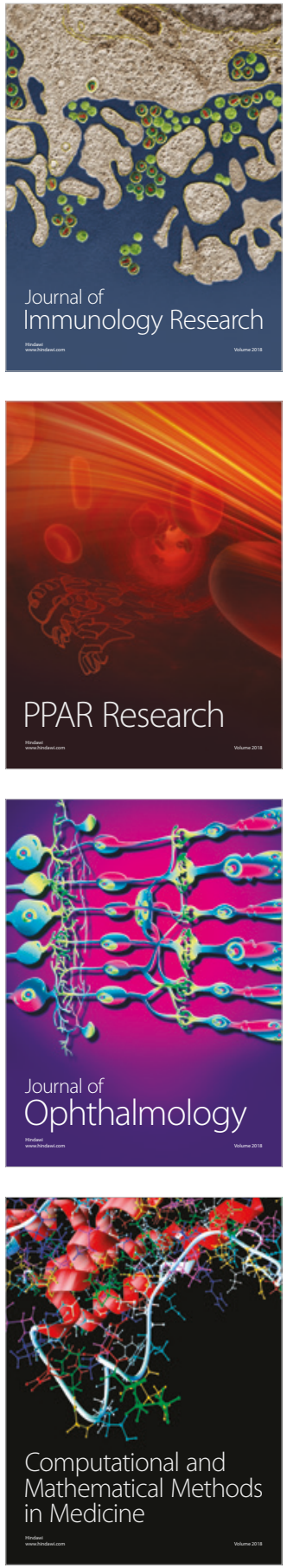

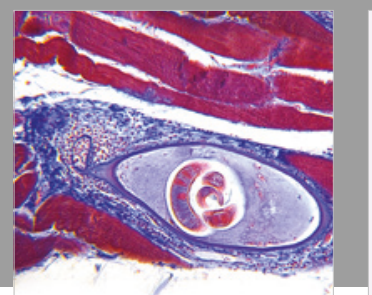

Gastroenterology Research and Practice

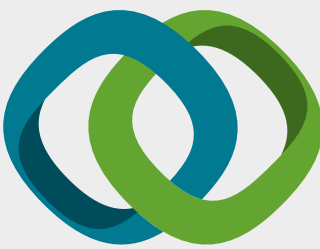

\section{Hindawi}

Submit your manuscripts at

www.hindawi.com
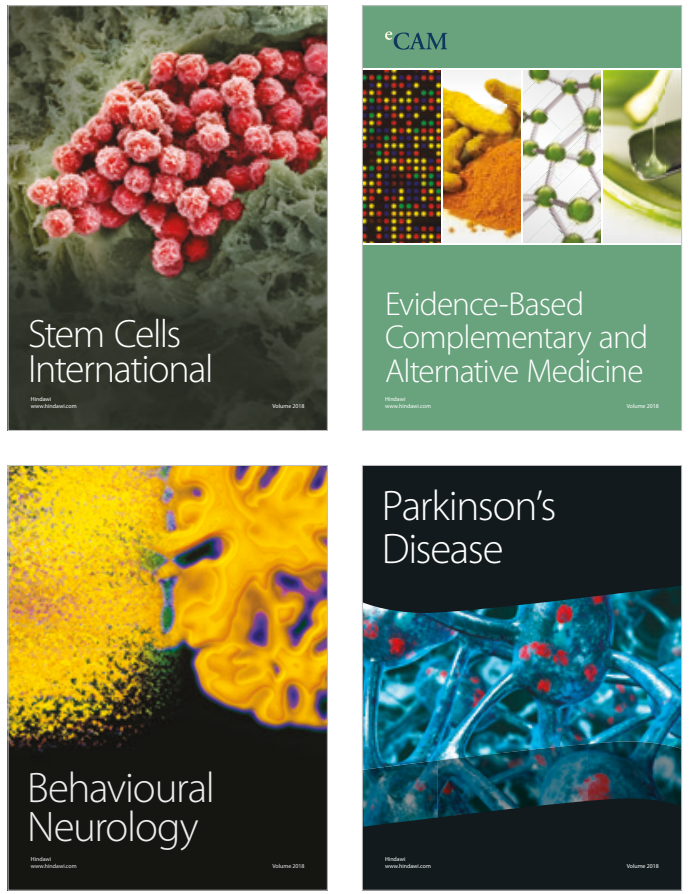

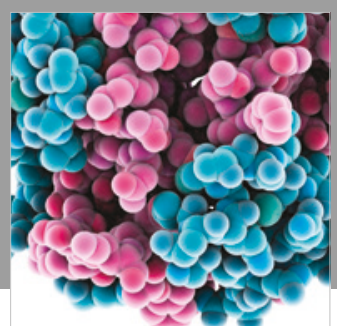

ournal of

Diabetes Research

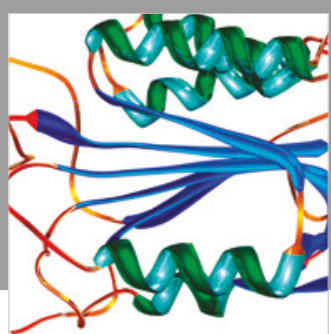

Disease Markers
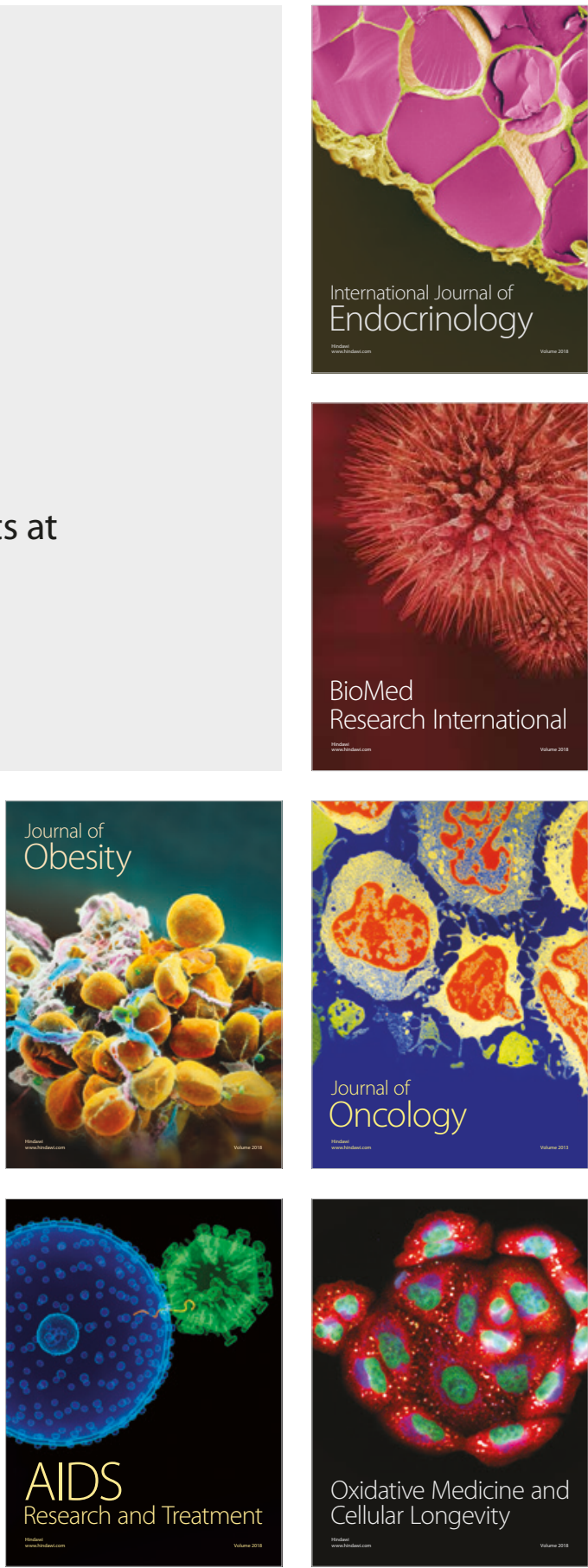